\section{Bei Ovarialkarzinomen den Beginn der Chemotherapie nicht zu lange hinauszögern}

\author{
Wissenschaftler untersuchten, ob die Zeit zwischen Operation und Beginn \\ der Chemotherapie einen Einfluss auf das Überleben von Patienten mit \\ fortgeschrittenem Ovarialkarzinom hat.
}

$\mathrm{D}$ as Intervall zwischen Operation und Beginn der Chemotherapie könnte für das Überleben von Frauen mit neu diagnostiziertem Ovarialkarzinom entscheidend sein. Eine Metaanalyse hatte gezeigt, dass das Rezidivrisiko steigt und das Gesamtüberleben sinkt, wenn sich der Beginn der Chemotherapie nach der Operation verzögert. Dieser Aspekt wurde nun in einer nachträglich durchgeführten Ad-hoc-Analyse der PhaseIII-Studie 218 der Gynecologic Oncology Group erneut untersucht.

1.718 Patienten mit neu diagnostiziertem fortgeschrittenem Ovarialkarzinom (Stadium III oder IV) wurden in diese Analyse einbezogen, darunter solche mit vollständiger Resektion, einer Resterkrankung mit geringem Volumen $(<1 \mathrm{~cm})$ und suboptimaler Resek- tion $(>1 \mathrm{~cm})$. Bei Patienten im Stadium III galt die Voraussetzung, dass eine makroskopische oder palpable Resterkrankung nach der Operation vorliegen musste. Eigentliches Ziel dieser Studie war es, den Nutzen des antiangiogenetischen Wirkstoffs Bevacizumab zusätzlich zur Chemotherapie in der Induktions- und Erhaltungstherapie zu untersuchen.

Die mediane Zeit zwischen Operation und Beginn der Chemotherapie betrug 31 Tage, bei $27 \%$ der Patienten waren es mehr als 40 Tage. Der Beginn der Chemotherapie nach weniger als 25 Tagen war nicht mit einem erhöhten Sterberisiko assoziiert. Nach 25 Tagen schien dieses Risiko jedoch deutlich zu steigen. In der multivariaten Analyse war die Zeit bis zum Beginn der Chemotherapie prädiktiv für das Gesamtüberleben ( $\mathrm{p}<$ 0,001 ), wobei die Gruppe mit vollständiger Resektion (Stadium IV) ein erhöhtes Sterberisiko hatte, wenn die Zeit bis zum Beginn der Chemotherapie 25 Tage überstieg. Beispielsweise stieg das Sterberisiko bei weißen Patienten mit vollständiger Resektion nach 25 Tagen um $27 \%$ für jede Verlängerung des Intervalls um $10 \%$. Am stärksten war der Einfluss des Zeitintervalls auf das Überleben bei Frauen mit mikroskopischer Resterkrankung.

Fazit: Das Überleben von Frauen mit fortgeschrittenem Ovarialkarzinom im Stadium IV könnte negativ beeinflusst werden, wenn die Chemotherapie erst mehr als 25 Tage nach der Operation beginnt. Weitere Studien sollten mögliche Gründe, die das Intervall zwischen Operation und Beginn der Chemotherapie beeinflussen, prospektiv untersuchen.

Judith Neumaier

Tewari KS et al. Early Initiation of Chemotherapy following Complete Resection of Advanced Ovarian Cancer Associated with Improved Survival: An NRG Oncology/Gynecologic Oncology Group Study. Ann Oncol. 2016 Jan;27(1):114-21

\section{Mammakarzinom: Trastuzumab bleibt Therapie der Wahl, auch nach Progression}

\begin{abstract}
Frauen mit metastasiertem HER2-positivem Brustkrebs, der unter oder kurz nach einer Trastuzumabtherapie fortgeschritten ist, profitieren nicht von der Umstellung der Behandlung auf Afatinib. Der Tyrosinkinaseinhibitor könnte prognostisch sogar von Nachteil sein.
\end{abstract}

W arum HER-2-positive Mammakarzinome unter Trastuzumab eine Resistenz gegen den Antikörper ausbilden, ist unklar. Vermutlich führt eine längerfristige Behandlung zur vermehrten EGFR- und HER3-Expression, ferner ist die Ausbildung von Heterodimeren zwischen HER2 und HER3 bedeutsam. Um die Resistenz zu überwinden, könnte es daher sinnvoll sein, Wirkstoffe mit einer breiteren Hemmung von ErbB-Rezeptoren einzusetzen. Diese Hypothese hat ein internationales Team um Nadia Harbeck, München, in der Studie LUXBreast 1 geprüft. 508 Frauen aus 41 Län- dern mit metastasiertem HER2-positivem Mammakarzinom, bei denen es unter einer Trastuzumabtherapie zur Progression gekommen war, wurden randomisiert (2:1) einer Behandlung mit Afatinib oder Trastuzumab, jeweils in Kombination mit Vinorelbin, unterzogen. Der Tyrosinkinasehemmer (TKI) Afatinib blockiert irreversibel die Signalleitung aller Homo- und Heterodimere der ErbB-Familie.

Die Aufnahme von Patientinnen in die Studie wurde wegen eines ungünstigen Nutzen-Risiko-Verhältnisse unter Afatinib/Vinorelbin vorzeitig gestoppt.
Die betroffenen Patientinnen mussten mit einer der Substanzen allein oder mit Trastuzumab plus Vinorelbin oder außerhalb der Studie weiterbehandelt werden. Für das progressionsfreie Überleben, den primären Studienendpunkt, war es unerheblich, welcher Gruppe die Patientinnen angehörten. Nach einem medianen Follow-up von 9,3 Monaten hatten $38 \%$ (Afatinib) bzw. $43 \%$ (Trastuzumab) ohne Progression überlebt. Das mediane progressionsfreie Überleben betrug 5,5 bzw. 5,6 Monate, ein nicht signifikanter Unterschied. Ein objektives Ansprechen - vollständig oder partiell - hatten $46 \%$ bzw. $47 \%$. Beim Gesamtüberleben schnitt die Afatinibgruppe dagegen mit 19,6 im Vergleich zu 28,6 Monaten unter Trastuzumab deutlich schlechter ab (mediane Beobachtungszeit elf Monate). Diese Ergebnisse können allerdings durch die Anschlussbehandlungen beeinflusst worden sein.

Unter Afatinib gab es zudem mehr Nebenwirkungen. $36 \%$ (vs. $26 \%$ unter 